\title{
Gibbs phenomenon for $p$-ary subdivision schemes
}

Jie Zhou ${ }^{1 *}$, Hongchan Zheng $^{1}$ and Baoxing Zhang ${ }^{1}$

"Correspondence:

zhjie@mail.nwpu.edu.cn

'Department of Applied

Mathematics, Northwestern

Polytechnical University, Xi'an, P.R. China

\begin{abstract}
When a Fourier series is used to approximate a function with a jump discontinuity, the Gibbs phenomenon always exists. This similar phenomenon exists for wavelets expansions. Based on the Gibbs phenomenon of a Fourier series and wavelet expansions of a function with a jump discontinuity, in this paper, we consider that a Gibbs phenomenon occurs for the $p$-ary subdivision schemes. Similar to the method of (Appl. Math. Lett. 76:157-163, 2018), we generalize the results about the stationary binary subdivision schemes in (Appl. Math. Lett. 76:157-163, 2018) to the case of $p$-ary subdivision schemes. By considering the masks of subdivision schemes, we obtain a sufficient condition to determine whether there exists a Gibbs phenomenon for $p$-ary subdivision schemes in the limit function close to the discontinuous point. This condition consists of the positivity of the partial sums of the values of the masks. By applying this condition, we can avoid the Gibbs phenomenon for $p$-ary subdivision schemes near discontinuity points. Finally, some examples in classical subdivision schemes are given to illustrate the results in this paper.
\end{abstract}

Keywords: Gibbs phenomenon; $p$-ary subdivision schemes; Wavelet expansions; Fourier series

\section{Springer}

\section{Introduction}

The Gibbs phenomenon means an overshoot of the approximation level for the partial sum of the Fourier series occurring at a simple discontinuity. It is well known that given an arbitrary continuous and periodic function $f(x)$, it is possible to represent it as a Fourier series. However, when a Fourier series is used to approximate a discontinuous or nonperiodic function, an overshoot occurs at the discontinuity. This phenomenon is known as the Gibbs effect. The phenomenon was noticed by Michelson and further explained by Gibbs [2, 3].

To illustrate what is happening in the Gibbs phenomenon, let us examine the partial sums of a Fourier series. Let $f(x)$ be a 2-periodic function defined by

$$
f(x)= \begin{cases}-1 & \text { for }-1 \leq x<0 \\ 1 & \text { for } 0 \leq x<1\end{cases}
$$

Let $s_{n}(x)$ be the $n$th partial sum of $f(x)$, then, for all $0<x_{1}<1$, the partial sums of $f(x)$ at $x_{1}$ approach $f\left(x_{1}\right)$. However, if $x$ is allowed to approach the discontinuity $0, s_{n}(x)$ converges

(c) The Author(s) 2019. This article is distributed under the terms of the Creative Commons Attribution 4.0 International License (http://creativecommons.org/licenses/by/4.0/), which permits unrestricted use, distribution, and reproduction in any medium, provided you give appropriate credit to the original author(s) and the source, provide a link to the Creative Commons license, and indicate if changes were made. 
to a number greater than 1 due to

$$
\lim _{n \rightarrow \infty} s_{n}(\pi / 2 n)=\frac{2}{\pi} \int_{0}^{\pi} \frac{\sin t}{t} d t=1.17898 \ldots>1 .
$$

See $[2,4,5]$ for many references therein and further details of this phenomenon on Fourier series.

An analogue of the Gibbs phenomenon is shown in wavelet expansions. Let $\phi$ be a compactly supported orthogonal scaling function generating a multiresolution analysis (MRA) $\left\{V_{k}\right\}$ for $L^{2}(\mathbb{R})$. For a given $f(x)$ in $L^{2}(\mathbb{R})$, the orthogonal projections $P_{k} f$ of $f$ onto the spaces $V_{k}$ are given by $P_{k} f=\sum_{n \in \mathbb{Z}}\left\langle f, \phi_{k, n}\right\rangle \phi_{k, n} . P_{k} f$ converge to $f$ in the $L^{2}(\mathbb{R})$ norm as $k \rightarrow \infty$. They form partial sums of the wavelets expansion of $f$ associated with the given scaling function $\phi$. Shim and Volkmer in [6] used a simple example to illustrate the existence of the Gibbs phenomenon in wavelet expansions. That is, let the scaling function be Shannon scaling function, $f$ be a square integrable bounded function with a jump discontinuity, there exists a Gibbs phenomenon at discontinuous points. The Gibbs phenomenon for wavelets expansions is further studied in [6-13]. In 1991, Richards [7] showed a Gibbs phenomenon for periodic spline functions. By considering the multiresolution analysis, the Gibbs function can be expressed in terms of the scaling function. By considering the size of the wavelet kernel, Kelly [8] gave a necessary and sufficient condition to prove existence of the Gibbs phenomenon for some compactly supported wavelets. Karanikas [9] described this phenomenon in continuous wavelet analysis. Ruch and Fleet studied this phenomenon for scaling vectors and illustrated that via positive scaling vectors exhibit no Gibbs phenomenon in [10]. For the periodic wavelet frame series, Zhang analyzed integral representations of the partial sums, convergence, and the Gibbs phenomenon of this situation in [12].

Subdivision scheme is an efficient tool for the approximation of a given function. For convergent schemes starting from discrete sets of control points and using basic rules of low complexity, curves or surfaces can be obtained as limits of sequences of points generated by recursive application of the subdivision scheme. The question has arisen as to whether there exists a Gibbs phenomenon in the limit functions close to a discontinuity point. The analysis and study of the Gibbs phenomenon is basically based on wavelet expansions and Fourier series. For subdivision schemes, there is little work about this phenomenon. Amat et al. [14] studied a new nonlinear dyadic subdivision scheme and briefly analyzed the Gibbs phenomenon that could occur in the limit function coming from the sampling of discontinuous function. Amat et al. [1] obtained a sufficient condition to analyze the Gibbs phenomenon for a stationary binary subdivision scheme. It is natural to ask whether we can derive the existence of the Gibbs phenomenon for other types subdivision schemes. This motivates us to further study the Gibbs phenomenon for the subdivision schemes.

However, in several applications of signal analysis [15-18], especially in digital image processing, the existence of this phenomenon is considered to be a disadvantage and leads to informational distortion. There have been some attempts to remove this phenomenon [19-23]. It looks back at Fejér averaging of the essentials of the summability theory, which is of importance to one of the basic methods of removing the effect of the Gibbs phenomenon. Also, there are the Lanczos averaging filter and Vandeven filter [19, 20]. By 
adding the Fourier coefficient of a Heaviside function, overshooting and distortional approximation near a point of discontinuity can be avoided in [21].

In this paper, we are concerned with the analysis of the Gibbs phenomenon for $p$-ary subdivision schemes. Based on the result of Amat in [1], we shall study the Gibbs phenomenon for $p$-ary subdivision schemes in the limit functions close to discontinuity zones. By analyzing the properties of the masks of the subdivision schemes, we present a sufficient condition to determine whether the Gibbs phenomenon occurs for the $p$-ary subdivision schemes near the discontinuous point. We also provide some numerical examples to validate our results.

The outline of the remainder of this paper is an follows. In Section 2, we introduce some basic definitions and facts about subdivision schemes and the Gibbs phenomenon. In Section 3, we obtain a sufficient condition to determine whether the Gibbs phenomenon occurs in $p$-ary subdivision schemes in the limit functions close to discontinuity zones. Section 4 provides some examples to validate our results. Section 5 concludes this paper.

\section{Preliminaries}

In this section, we recall some basic definitions and known facts about subdivision schemes and the Gibbs phenomenon, which form the basis of the rest of this paper.

Given a sequence of initial control points $f^{0}=\left\{f_{i}^{0}: i \in \mathbb{Z}\right\}$, the new control points $f^{k+1}=$ $\left\{f_{i}^{k+1}: i \in \mathbb{Z}\right\}$ are iteratively refined with the refinement rule

$$
\left(S_{a} f^{k}\right)_{i}=f_{i}^{k+1}=\sum_{j \in \mathbb{Z}} a_{i-p j} f_{j}^{k}, \quad i \in \mathbb{Z}
$$

where the integer $p \geq 2$ is called arity, $p=2$ is called binary subdivision scheme, and the subdivision scheme is denoted by $S_{a}$. The sequence $a=\left\{a_{j}: j \in \mathbb{Z}\right\}$ is called subdivision mask. Generally speaking, the masks are always supposed to be finitely supported. The limit curve is uniquely determined by the initial control points and subdivision mask $a$.

For a given subdivision $S_{a}$, the vertexes $f_{p i+l}^{k+1}$ are also calculated with

$$
\left(S_{a} f^{k}\right)_{p i+l}=f_{p i+l}^{k+1}=\sum_{\gamma \in \mathbb{Z}} a_{p \gamma+l} f_{i-\gamma}^{k}, \quad i \in \mathbb{Z}
$$

for $0 \leq l \leq p-1$.

The symbol of a subdivision scheme $S_{a}$ is given by

$$
a(z)=\sum_{j \in \mathbb{Z}} a_{j} z^{j}
$$

which is an important tool to study the properties of subdivision schemes. Set $a^{[k]}$ be the $k$ iterated symbol, it is known that $a^{[k]}(z)=\prod_{l=1}^{k} a\left(z^{p^{l-1}}\right)$ [24]. For $0 \leq l<p^{k}$, we have

$$
\begin{aligned}
f_{p^{k} i+l}^{k} & =\left(S_{a} f^{k-1}\right)_{p^{k i+l}}=\left(S_{a} S_{a} f^{k-2}\right)_{p^{k} i+l}=\cdots \\
& =\left(S_{a}^{k} f^{0}\right)_{p^{k} i+l}=\left(S_{a^{[k}} f^{0}\right)_{p^{k} i+l}=\sum_{\gamma \in \mathbb{Z}} a_{p^{k} \gamma+l}^{[k]} f_{i-\gamma}^{0},
\end{aligned}
$$


with

$$
a_{j}^{[k]}=\sum_{i \in \mathbb{Z}} a_{i}^{[k-1]} a_{j-p i}
$$

where $a_{j}^{[1]}=a_{j}$. Throughout this paper, we require the mask $a=\left\{a_{j}: j \in \mathbb{Z}\right\}$ satisfying

$$
\begin{cases}-1 \leq a_{j} \leq 1, & M \leq j \leq M+N, \\ a_{j}=0, & j>M+N \text { or } j<M, \\ a_{M}, & a_{M+N} \neq 0,\end{cases}
$$

where $M<0$ and $N$ are fixed integers with $N \geq 2$ and $M+N>0$.

By attaching $f_{i}^{k}$ to $\frac{i}{p^{k}}$ for $i \in \mathbb{Z}, k \geq 0$, we define the notion of convergence of the subdivision scheme. We say a stationary subdivision scheme $S_{a}$ converges to a function $f \in C^{0}$, for any bounded initial control sequence $f^{0}=\left\{f_{i}^{0}, i \in \mathbb{Z}\right\}$, if there exists a continuous function $f$ such that

$$
\lim _{k \rightarrow \infty}\left\|f\left(\frac{i}{p^{k}}\right)-f_{i}^{k}\right\|_{\infty}=0
$$

denote $S_{a}^{\infty} f^{0}=f$. In this case, we say the scheme $S_{a}$ is $C^{0}$ convergent. We restrict our discussion to non-singular schemes for which $S_{a}^{\infty} f^{0} \equiv 0$ if and only if $f^{0}=0$. Next, we review a necessary condition for the convergence of the subdivision scheme.

Theorem 2.1 If a subdivision scheme $S_{a}$ is convergent, then the mask a of this subdivision scheme satisfies

$$
\sum_{\gamma \in Z} a_{p \gamma+l}=1, \quad l=0,1, \ldots, p-1
$$

We recall the notion of parametrization which can be used to introduce the Gibbs phenomenon in the subdivision schemes. Usually, set

$$
t_{i}^{(k)}:=p^{-k}(i+c) h, \quad i \in \mathbb{Z}, c \in \mathbb{R}, k \geq 0, h>0 .
$$

Following [25, 26], the parametrization (2.8) is called primal when $c=0$ and dual when $c=-\frac{1}{2}$. It is well known that most of the properties of a subdivision scheme (e.g., convergence, generation, smoothness, or support size) do not depend on the choice of the parameter values $t_{i}^{(k)}$. However, with respect to the subdivision capability of reproducing specific classes of functions, $t_{i}^{(k)}$ plays a very important role. By choosing suitable $c$, the reproduction property of subdivision schemes can be improved and achieve the best.

An important issue in the implementation of subdivision algorithm is how to guarantee the approximation error to be as small as possible. The high quality algorithm is usually determined by the order of the approximation. Suppose that the given initial data $f^{0}$ is of the form $f^{0}:=\left\{f_{i}^{0}\right\}_{i \in \mathbb{Z}}$ with a smooth function $f$. Then our concern is to find the largest exponent $m>0$ such that

$$
\left\|f^{\infty}-f\right\|_{L_{\infty}(\Omega)} \leq B^{\prime} h^{m}
$$


with a constant $B^{\prime}>0$, where $\Omega$ is a compact set in $R$. The exponent $m$ is called the approximation order of the subdivision scheme.

In this paper, we will impose on the scheme $S_{a}$ satisfying the following condition: C1. For any $f \in C^{n}, n \geq 2$, and $h>0$, with the initial data $f_{i}^{0}=f(i h)\left(\right.$ or $\left.f\left(\left(i-\frac{1}{2}\right) h\right)\right), i \in \mathbb{Z}$, then

$$
\max _{x \in \mathbb{R}}\left|\left(S_{a}^{\infty} f^{0}\right)(x)-f(x)\right| \leq B h^{n},
$$

where $B$ is a constant which depends on $f$, but it does not depend on $h$. In the following, we give a precise definition of what we mean by the Gibbs phenomenon for the subdivision schemes.

Definition 2.1 ([1]) For a given discontinuous function $f(x)$ with a jump discontinuity point $\xi$, its sampling $f_{h}$ defined by $f_{i, h}=f(i h)$ (or $f_{i, h}=f\left(\left(i-\frac{1}{2}\right) h\right)$ ), let the subdivision scheme $S_{a}$ be convergent, the Gibbs phenomenon can be delimited by the following two properties:

1. For any point $x$ away from the discontinuity $\xi$, the convergence of $\left(S_{a}^{\infty} f_{h}\right)$ towards $f$ is rather slow, that is,

$$
\left|f(x)-\left(S_{a}^{\infty} f_{h}\right)(x)\right|=O(h)
$$

2. For any point $x$ close to the discontinuity $\xi$, there is an overshoot, that is,

$$
\max _{x \in \mathbb{R}}\left|f(x)-\left(S_{a}^{\infty} f_{h}\right)(x)\right|
$$

does not tend to zero when $h$ goes to 0 .

In fact, for any discontinuous function $f$ with a jump discontinuity point $\xi$, the Gibbs phenomenon is related to the convergence of $\left(S_{a}^{\infty} f_{h}\right)$ towards $f$ when $h$ goes to 0 .

\section{Main results}

In this section, we focus on the Gibbs phenomenon for $p$-ary subdivision schemes. By directly considering the masks of $p$-ary subdivision schemes, we present the following theorem to obtain the sufficient conditions to avoid the Gibbs phenomenon in $p$-ary subdivision schemes.

Theorem 3.1 For given $\xi(0 \leq \xi \leq h)$, let $f$ be any discontinuous function with a jump discontinuity point $\xi$ defined by

$$
f(x)=\left\{\begin{array}{lll}
f_{-}(x), & \left.\left.f_{-} \in C^{n}(]-\infty, \xi\right]\right), & \forall x \leq \xi, \\
f_{+}(x), & f_{+} \in C^{n}([\xi,+\infty[), & \forall x>\xi
\end{array}\right.
$$

with $n \geq 2$ and $f_{-}(\xi)>f_{+}(\xi)$. Let $S_{a}$ be a convergent $p$-ary subdivision scheme with mask a satisfying condition (2.5), and $h$ be a sufficiently small positive number. We have

(1). If $|x| \geq \max \left\{\left|\frac{1}{p-1}(M-p+1)\right|,\left|\frac{1}{p-1}(M+N)+1\right|\right\} h$, then

$$
\left|f(x)-\left(S_{a}^{\infty} f_{h}\right)(x)\right|=O\left(h^{n}\right)
$$

with $n \geq 2$. 
(2). If $|x| \leq \max \left\{\left|\frac{1}{p-1}(M-p+1)\right|,\left|\frac{1}{p-1}(M+N)+1\right|\right\} h$, let

$$
\lambda_{l}^{[k]}(i)= \begin{cases}\sum_{\tau \leq i} a_{p^{k} \tau+l}^{[k]}, & i<0, \\ 0, & i=0, \\ \sum_{\tau \geq i} a_{p^{k} \tau+l}^{[k]}, & i>0,\end{cases}
$$

$0 \leq l<p^{k}$, where $a^{[k]}$ is defined in Eq. (2.4). If $\lambda_{l}^{[k]}(i) \geq 0, \forall i, k$, then there exist $\alpha_{h}=O(h)$ such that

$$
f_{1, h}-\alpha_{h} \leq f_{+}(h)-\alpha_{h} \leq\left(S_{a}^{\infty} f_{h}\right)(x) \leq f_{-}(0)+\alpha_{h}=f_{0, h}+\alpha_{h}
$$

Proof For any iteration $k$, there exist $P_{k}^{-}, P_{k}^{+}$such that, for all $i \notin\left[P_{k}^{-}, P_{k}^{+}\right]$, the evaluation $\left(S_{a}^{k+1} f_{h}\right)_{p i+l}, 0 \leq l<p$ is applied starting from regular data, that is, $i \notin\left[P_{k}^{-}, P_{k}^{+}\right]$, the Gibbs phenomenon does not appear. So, we only consider the Gibbs phenomenon in $i \in\left[P_{k}^{-}, P_{k}^{+}\right]$.

(1). Let us firstly consider $k=0$. As $0 \leq \xi \leq h$, the discontinuity is between the values $f_{0, h}$ and $f_{1, h}$, then by Eq. (2.2), we have $i-\gamma=0$ and $i-\gamma=1$, these values are used. We only have to determine the values $a_{p i+l}, a_{p(i-1)+l}, 0 \leq l<p$. By considering the condition of the mask $a$ in (2.5), we have

$$
P_{0}^{-}=\frac{M-p+1}{p}, \quad P_{0}^{+}=\frac{M+N+p}{p}
$$

For $k=1$, the points calculated using $f_{0, h}$ and $f_{1, h}$ at level $k=1$ are $i \in\left[P_{0}^{-}, P_{0}^{+}\right]$. Because

$$
\left(S_{a} f\right)_{p i+l}=\sum_{\gamma \in \mathbb{Z}} a_{p \gamma+l} f_{i-\gamma, h}=\sum_{\gamma \in \mathbb{Z}} a_{p \gamma+l}\left(S_{a} f_{h}^{0}\right)_{i-\gamma, h}
$$

so, $p i+l \in\left[p P_{0}^{-}, p P_{0}^{+}\right]$, that is, $i-\gamma \in\left[p P_{0}^{-}, p P_{0}^{+}\right], \gamma \in\left[i-p P_{0}^{+}, i-p P_{0}^{-}\right]$. Thus we have

$$
\left\{\begin{array}{l}
p \gamma \in\left[p i-p^{2} P_{0}^{+}, p i-p^{2} P_{0}^{-}\right], \\
p \gamma+1 \in\left[p i-p^{2} P_{0}^{+}+1, p i-p^{2} P_{0}^{-}+1\right], \\
\cdots \\
p \gamma+p-1 \in\left[p i-p^{2} P_{0}^{+}+p-1, p i-p^{2} P_{0}^{-}+p-1\right] .
\end{array}\right.
$$

By the condition of the mask $a$ in (2.5), we have

$$
P_{1}^{-}=\frac{M+p^{2} P_{0}^{-}-p+1}{p}=p P_{0}^{-}+P_{0}^{-}, \quad P_{1}^{+}=\frac{M+N+p^{2} P_{0}^{+}}{p}
$$

By induction, we have

$$
\begin{aligned}
P_{k}^{-} & =P_{0}^{-}+p P_{k-1}^{-} \\
& =P_{0}^{-}+p\left(P_{0}^{-}+p P_{k-2}^{-}\right)=P_{0}^{-}+p P_{0}^{-}+p^{2} P_{k-2}^{-} \\
& =\cdots=P_{0}^{-}+p P_{0}^{-}+p^{2} P_{0}^{-}+\cdots+p^{k} P_{0}^{-} \\
& =\frac{1}{p-1}\left(p^{k+1}-1\right) \frac{M-p+1}{p} .
\end{aligned}
$$


By using the same method, we have

$$
P_{k}^{+}=\frac{1}{p-1}\left(p^{k+1}-1\right) \frac{M+N}{p}+p^{k} .
$$

$\forall i \in\left[P_{k}^{-}, P_{k}^{+}\right]$, considering primal parametrization of the $p$-ary subdivision scheme in (2.8). Then, for condition (C1) and

$$
|x| \geq \max \left\{\left|\frac{1}{p-1}(M-p+1)\right|,\left|\frac{1}{p-1}(M+N)+1\right|\right\} h,
$$

we have

$$
\left|f(x)-\left(S_{a}^{\infty} f_{h}\right)(x)\right|=O\left(h^{n}\right)
$$

with $n \geq 2$. Next, we analyze the Gibbs phenomenon for a $p$-ary subdivision scheme satisfying

$$
|x| \leq \max \left\{\left|\frac{1}{p-1}(M-p+1)\right|,\left|\frac{1}{p-1}(M+N)+1\right|\right\} h .
$$

(2). Analogous to the proof of (1), we consider an induction process to prove this part.

For $k=1$,

$$
\begin{aligned}
& \left(S_{a} f_{h}\right)_{p i}=\sum_{\gamma \in \mathbb{Z}} a_{p \gamma} f_{i-\gamma}=a_{0} f_{i, h}+\sum_{\gamma \leq-1} a_{p \gamma} f_{i-\gamma, h}+\sum_{\gamma \geq 1} a_{p \gamma} f_{i-\gamma, h} \\
& =f_{i, h}-\left(\sum_{\tau \leq-1} a_{p \tau}+\sum_{\tau \geq 1} a_{p \tau}\right) f_{i, h}+\left[a_{-p} f_{i+1, h}+a_{-2 p} f_{i+2, h}+\cdots+a_{-p \gamma} f_{i+\gamma, h}+\cdots\right] \\
& +\left[a_{p} f_{i-1, h}+a_{2 p} f_{i-2, h}+\cdots+a_{p \gamma} f_{i-\gamma, h}+\cdots\right] \\
& =f_{i, h}-\left(\sum_{\tau \leq-1} a_{p \tau}+\sum_{\tau \geq 1} a_{p \tau}\right) f_{i, h}+\left[\sum_{\tau \leq-1} a_{p \tau} f_{i+1, h}-\sum_{\tau \leq-2} a_{p \tau} f_{i+1, h}\right. \\
& \left.+\sum_{\tau \leq-2} a_{p \tau} f_{i+2, h}-\sum_{\tau \leq-3} a_{p \tau} f_{i+2, h}+\cdots+\sum_{\tau \leq-\gamma} a_{p \tau} f_{i+\gamma, h}-\sum_{\tau \leq-\gamma-1} a_{p \tau} f_{i+\gamma, h}+\cdots\right] \\
& +\left[\sum_{\tau \geq 1} a_{p \tau} f_{i-1, h}-\sum_{\tau \geq 2} a_{p \tau} f_{i-1, h}+\sum_{\tau \geq 2} a_{p \tau} f_{i-2, h}-\sum_{\tau \geq 3} a_{p \tau} f_{i-2, h}+\cdots\right. \\
& \left.+\sum_{\tau \geq \gamma} a_{p \tau} f_{i-\gamma, h}-\sum_{\tau \geq \gamma+1} a_{p \tau} f_{i-\gamma, h}+\cdots\right] \\
& =f_{i, h}+\sum_{\gamma \leq-1}\left(\sum_{\tau \leq \gamma} a_{p \tau}\right)\left(f_{i-\gamma, h}-f_{i-\gamma-1, h}\right)+\sum_{\gamma \geq 1}\left(\sum_{\tau \geq \gamma} a_{p \tau}\right)\left(f_{i-\gamma, h}-f_{i-\gamma+1, h}\right) \\
& =f_{i, h}+\sum_{\gamma \leq-1} \lambda_{0}(\gamma)\left(f_{i-\gamma, h}-f_{i-\gamma-1, h}\right)+\sum_{\gamma \geq 1} \lambda_{0}(\gamma)\left(f_{i-\gamma, h}-f_{i-\gamma+1, h}\right) . \\
& \left(S_{a} f_{h}\right)_{p i+1}=\sum_{\gamma \in \mathbb{Z}} a_{p \gamma+1} f_{i-\gamma}=a_{1} f_{i, h}+\sum_{\gamma \leq-1} a_{p \gamma+1} f_{i-\gamma, h}+\sum_{\gamma \geq 1} a_{p \gamma+1} f_{i-\gamma, h} \\
& =f_{i, h}-\left(\sum_{\tau \leq-1} a_{p \tau+1}+\sum_{\tau \geq 1} a_{p \tau+1}\right) f_{i, h}+\left[a_{-p+1} f_{i+1, h}+a_{-2 p+1} f_{i+2, h}\right.
\end{aligned}
$$




$$
\begin{aligned}
& \left.+\cdots+a_{-p \gamma+1} f_{i+\gamma, h}+\cdots\right]+\left[a_{p+1} f_{i-1, h}+a_{2 p+1} f_{i-2, h}+\cdots\right. \\
& \left.+a_{p \gamma+1} f_{i-\gamma, h}+\cdots\right] \\
= & f_{i, h}-\left(\sum_{\tau \leq-1} a_{p \tau+1}+\sum_{\tau \geq 1} a_{p \tau+1}\right) f_{i, h}+\left[\sum_{\tau \leq-1} a_{p \tau+1} f_{i+1, h}-\sum_{\tau \leq-2} a_{p \tau+1} f_{i+1, h}\right. \\
& +\sum_{\tau \leq-2} a_{p \tau+1} f_{i+2, h}-\sum_{\tau \leq-3} a_{p \tau+1} f_{i+2, h}+\cdots+\sum_{\tau \leq-\gamma} a_{p \tau+1} f_{i+\gamma, h} \\
& \left.-\sum_{\tau \leq-\gamma-1} a_{p \tau+1} f_{i+\gamma, h}+\cdots\right] \\
& +\left[\sum_{\tau \geq 1} a_{p \tau+1} f_{i-1, h}-\sum_{\tau \geq 2} a_{p \tau+1} f_{i-1, h}+\sum_{\tau \geq 2} a_{p \tau+1} f_{i-2, h}-\sum_{\tau \geq 3} a_{p \tau+1} f_{i-2, h}+\cdots\right. \\
& \left.+\sum_{\tau \geq \gamma} a_{p \tau+1} f_{i-\gamma, h}-\sum_{\tau \geq \gamma+1} a_{p \tau+1} f_{i-\gamma, h}+\cdots\right] \\
= & f_{i, h}+\sum_{\gamma \leq-1}\left(\sum_{\tau \leq \gamma} a_{p \tau+1}\right)\left(f_{i-\gamma, h}-f_{i-\gamma-1, h}\right)+\sum_{\gamma \geq 1}\left(\sum_{\tau \geq \gamma} a_{p \tau+1}\right)\left(f_{i-\gamma, h}-f_{i-\gamma+1, h}\right) \\
= & f_{i, h}+\sum_{\gamma \leq-1} \lambda_{1}(\gamma)\left(f_{i-\gamma, h}-f_{i-\gamma-1, h}\right)+\sum_{\gamma \geq 1} \lambda_{1}(\gamma)\left(f_{i-\gamma, h}-f_{i-\gamma+1, h}\right) .
\end{aligned}
$$

Analogously, for $\left(S_{a} f_{h}\right)_{p i+2},\left(S_{a} f_{h}\right)_{p i+3}, \ldots,\left(S_{a} f_{h}\right)_{p i+(p-1)}$, we have

$$
\left(S_{a} f_{h}\right)_{p i+l}=f_{i, h}+\sum_{\gamma \leq-1} \lambda_{l}(\gamma)\left(f_{i-\gamma, h}-f_{i-\gamma-1, h}\right)+\sum_{\gamma \geq 1} \lambda_{l}(\gamma)\left(f_{i-\gamma, h}-f_{i-\gamma+1, h}\right),
$$

where

$$
\lambda_{l}(i)= \begin{cases}\sum_{\tau \leq i} a_{p \tau+l}, & i<0, \\ 0, & i=0, \\ \sum_{\tau \geq i} a_{p \tau+l}, & i>0, l=0,1, \ldots, p-1 .\end{cases}
$$

If $i \leq 0$, then

$$
\begin{aligned}
\left(S_{a} f_{h}\right)_{p i+l}= & f_{i, h}+\sum_{\gamma \leq-1} \lambda_{l}(\gamma)\left(f_{i-\gamma, h}-f_{i-\gamma-1, h}\right)+\sum_{\gamma \geq 1} \lambda_{l}(\gamma)\left(f_{i-\gamma, h}-f_{i-\gamma+1, h}\right) \\
= & f_{i, h}+\lambda_{l}(i-1)\left(f_{1, h}-f_{0, h}\right)+\sum_{\gamma \leq-1, \gamma \neq i-1} \lambda_{l}(\gamma)\left(f_{i-\gamma, h}-f_{i-\gamma-1, h}\right) \\
& +\sum_{\gamma \geq 1} \lambda_{l}(\gamma)\left(f_{i-\gamma, h}-f_{i-\gamma+1, h}\right) \\
= & f_{i, h}+\lambda_{l}(i-1)\left(f_{1, h}-f_{0, h}\right)+O(h) \\
= & f_{0, h}+\lambda_{l}(i-1)\left(f_{1, h}-f_{0, h}\right)+f_{i, h}-f_{0, h}+O(h) .
\end{aligned}
$$

If $i>0$, then

$$
\left(S_{a} f_{h}\right)_{p i+l}=f_{i, h}+\lambda_{l}(i)\left(f_{0, h}-f_{1, h}\right)+\sum_{\gamma \leq-1} \lambda_{l}(\gamma)\left(f_{i-\gamma, h}-f_{i-\gamma-1, h}\right)
$$




$$
\begin{aligned}
& +\sum_{\gamma \geq 1, \gamma \neq i} \lambda_{l}(\gamma)\left(f_{i-\gamma, h}-f_{i-\gamma+1, h}\right) \\
= & f_{i, h}+\lambda_{l}(i)\left(f_{0, h}-f_{1, h}\right)+O(h) \\
= & f_{1, h}+\lambda_{l}(i)\left(f_{0, h}-f_{1, h}\right)+f_{i, h}-f_{1, h}+O(h) .
\end{aligned}
$$

Since $\lambda_{l}(i) \geq 0, \forall i$, and $f_{0, h}-f_{1, h}>0$, we obtain that if $i \in\left[P_{1}^{-}, P_{1}^{+}\right]$,

$$
f_{1, h}-O(h) \leq\left(S_{a} f_{h}\right)_{p i+l} \leq f_{0, h}+O(h) .
$$

If $k=2$, according to (2.3), we get

$$
f_{p^{2} i+l}^{2}=\left(S_{a}^{2} f^{0}\right)_{p^{2} i+l}=\left(S_{a^{[2]}} f^{0}\right)_{p^{2} i+l}=\sum_{\gamma \in \mathbb{Z}} a_{p^{2} \gamma+l}^{[2]} f_{i-\gamma}^{0} .
$$

When $l=0$, by applying (2.4), we obtain

$$
a_{p^{2} \tau}^{[2]}=\sum_{i \in \mathbb{Z}} a_{i} a_{p^{2} \tau-p i}
$$

Let $p^{2} \tau-p i=p j$, we have

$$
\begin{aligned}
\sum_{\tau \in \mathbb{Z}} a_{p^{2} \tau}^{[2]}= & a_{0}^{[2]}+\sum_{\tau \leq-1} a_{p^{2} \tau}^{[2]}+\sum_{\tau \geq 1} a_{p^{2} \tau}^{[2]}=\sum_{\tau \in \mathbb{Z}} a_{p \tau-j} \sum_{j \in \mathbb{Z}} a_{p j}=1, \\
\left(S_{a}^{2} f_{h}\right)_{p^{2} i}= & a_{0}^{[2]} f_{i, h}+\sum_{\gamma \leq-1} a_{p^{2} \gamma}^{[2]} f_{i-\gamma, h}+\sum_{\gamma \geq 1} a_{p^{2} \gamma}^{[2]} f_{i-\gamma, h} \\
= & f_{i, h}\left(1-\sum_{\tau \geq 1} a_{p^{2} \tau}^{[2]}-\sum_{\tau \leq-1} a_{p^{2} \tau}^{[2]}\right)+\left(a_{-p^{2}}^{[2]} f_{i+1, h}+a_{-2 p^{2}}^{[2]} f_{i+2, h}+\cdots\right. \\
& \left.+a_{\gamma p^{2}}^{[2]} f_{i-\gamma, h}+\cdots\right)+\left(a_{p^{2}}^{[2]} f_{i-1, h}+a_{2 p^{2}}^{[2]} f_{i-2, h}+\cdots+a_{\gamma p^{2}}^{[2]} f_{i-\gamma, h}+\cdots\right) \\
= & f_{i, h}\left(1-\sum_{\tau \geq 1} a_{p^{2} \tau}^{[2]}-\sum_{\tau \leq-1} a_{p^{2} \tau}^{[2]}\right)+\left(\sum_{\tau \leq-1} a_{p^{2} \tau}^{[2]} f_{i+1, h}-\sum_{\tau \leq-2} a_{p^{2} \tau}^{[2]} f_{i+1, h}\right. \\
& +\sum_{\tau \leq-2} a_{p^{2} \tau}^{[2]} f_{i+2, h}-\sum_{\tau \leq-3} a_{p^{2} \tau}^{[2]} f_{i+2, h}+\cdots+\sum_{\tau \leq \gamma} a_{p^{2} \tau}^{[2]} f_{i-\gamma, h} \\
& \left.-\sum_{\tau \leq \gamma-1} a_{p^{2} \tau}^{[2]} f_{i-\gamma, h}+\cdots\right)+\left(\sum_{\tau \geq 1} a_{p^{2} \tau}^{[2]} f_{i-1, h}-\sum_{\tau \geq 2} a_{p^{2} \tau}^{[2]} f_{i-1, h}\right. \\
& +\sum_{\tau \geq 2} a_{p^{2} \tau}^{[2]} f_{i-2, h}-\sum_{\tau \geq 3} a_{p^{2} \tau}^{[2]} f_{i-2, h}+\cdots+\sum_{\tau \geq \gamma} a_{p^{2} \tau}^{[2]} f_{i-\gamma, h} \\
& \left.-\sum_{\tau \geq \gamma+1} a_{p^{2} \tau}^{[2]} f_{i-\gamma, h}+\cdots\right) \\
= & f_{i, h}+\sum_{\gamma \leq-1}\left(\sum_{\tau \leq \gamma} a_{p^{2} \tau}^{[2]}\right)\left(f_{i-\gamma, h}-f_{i-\gamma-1, h}\right)+\sum_{\gamma \geq 1}\left(\sum_{\tau \geq \gamma} a_{p^{2} \tau}^{[2]}\right)\left(f_{i-\gamma, h}-f_{i-\gamma+1, h}\right) \\
= & f_{i, h}+\sum_{\gamma \leq-1} \lambda_{0}^{[2]}(\gamma)\left(f_{i-\gamma, h}-f_{i-\gamma-1, h}\right)+\sum_{\gamma \geq 1} \lambda_{0}^{[2]}(\gamma)\left(f_{i-\gamma, h}-f_{i-\gamma+1, h}\right), \\
& \\
&
\end{aligned}
$$


where

$$
\lambda_{0}^{[2]}(i)= \begin{cases}\sum_{\tau \leq i} a_{p^{2} \tau}^{[2]}, & i<0, \\ 0, & i=0, \\ \sum_{\tau \geq i} a_{p^{2} \tau}^{[2]}, & i>0 .\end{cases}
$$

By using the analogous method, for $\left(S_{a}^{2} f_{h}\right)_{p^{2} i+1},\left(S_{a}^{2} f_{h}\right)_{p^{2} i+2}, \ldots\left(S_{a}^{2} f_{h}\right)_{p^{2} i+p-1}$, we have analogous results. So, for all $l, 0 \leq l \leq p-1$, if $\lambda_{l}^{[2]}(i) \geq 0$, we can obtain

$$
f_{1, h}-O(h) \leq\left(S_{a}^{2} f_{h}\right)_{p^{2} i+l} \leq f_{0, h}+O(h)
$$

For all $k$, by using an induction process, we have the following results.

If $\lambda_{l}^{[k]}(i) \geq 0, \forall i, k$, there exist $\alpha_{h}=O(h)$ such that

$$
f_{1, h}-\alpha_{h} \leq f_{+}(h)-\alpha_{h} \leq\left(S_{a}^{\infty} f_{h}\right)(x) \leq f_{-}(0)+\alpha_{h}=f_{0, h}+\alpha_{h} .
$$

This completes the proof of this theorem.

By analyzing the proof of Theorem 3.1, we can find that, for all $k$ and $i \in\left[P_{k}^{-}, P_{k}^{+}\right]$, if $\lambda_{l}^{[k]}(i) \geq$ $0, \forall i, k$, Eq. (3.2) is true. Because $\lambda_{l}^{[k]}(i)$ is related to the partial sums of the values of the masks. So we have the following corollary.

Corollary 3.1 Let $S_{a}$ be a convergent $p$-ary subdivision scheme with mask $a=\left\{a_{M}, \ldots\right.$, $\left.a_{M+N}\right\}$. If $a_{j}>0, \forall M \leq j \leq M+N$, then $S_{a}$ does not produce the Gibbs phenomenon in the limit function close to discontinuity zones.

In the next section, we will show four examples to demonstrate that our results are efficient.

\section{Example}

In this section, we show the Gibbs phenomena in four classical subdivision schemes to illustrate the results of this paper. We consider the limit function obtained in some typical subdivision schemes when the initial data is a sampling of the following function:

$$
g(x)= \begin{cases}1, & x \in[0,1] \\ 0, & x \in(1,2] \\ 1, & x \in(2,3]\end{cases}
$$

We take the binary 5-point approximating scheme in [27]:

$$
\left\{\begin{array}{l}
f_{2 i}^{k+1}=\frac{u}{128} f_{i-2}^{k}+\left(\frac{u}{32}+\frac{3}{16}\right) f_{i-1}^{k}+\left(\frac{5}{8}-\frac{5 u}{64}\right) f_{i}^{k}+\left(\frac{u}{32}+\frac{3}{16}\right) f_{i+1}^{k}+\frac{u}{128} f_{i+2}^{k} \\
f_{2 i+1}^{k+1}=\left(\frac{u}{32}+\frac{1}{32}\right) f_{i-1}^{k}+\left(\frac{15}{32}-\frac{u}{32}\right) f_{i}^{k}+\left(\frac{15}{32}-\frac{u}{32}\right) f_{i+1}^{k}+\left(\frac{u}{32}+\frac{1}{32}\right) f_{i+2}^{k} .
\end{array}\right.
$$

According to Corollary 3.1, because of the positivity of the masks of the binary 5-point scheme with $u=1$, this scheme does not produce the Gibbs phenomenon in the limit functions close to discontinuity zones. The result for this scheme is shown by Fig. 1(a). 


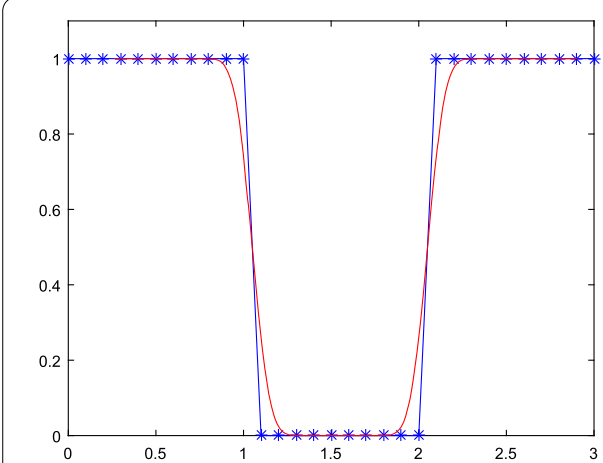

(a): binary 5 -point scheme

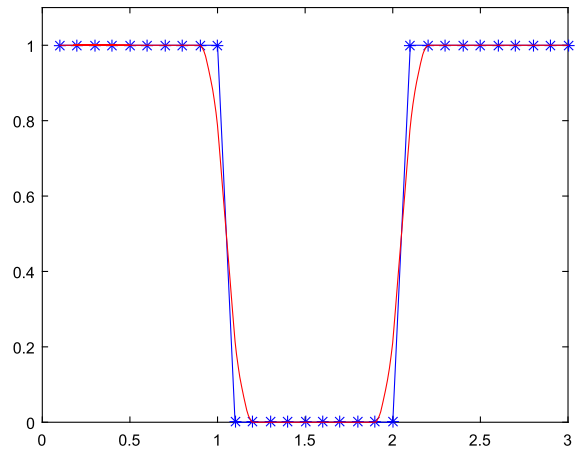

(c): ternary 3-point scheme

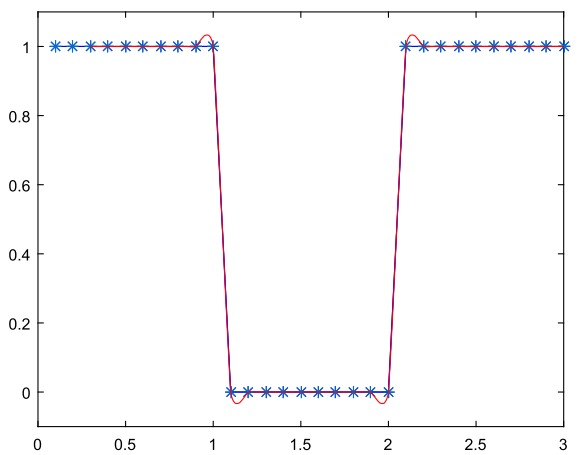

(b): D D's scheme with $\mathrm{m}=1$

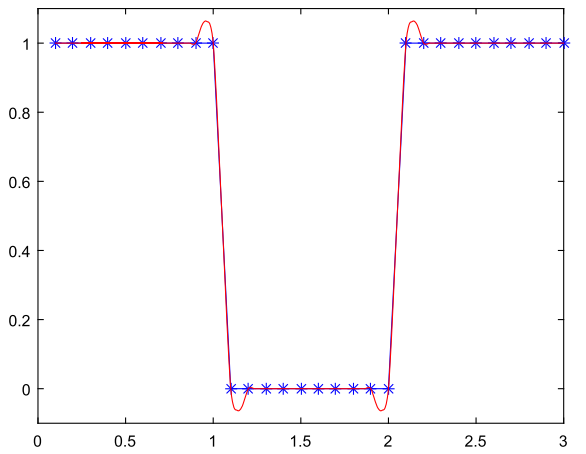

(d): ternary 5-point scheme

Figure 1 The limit functions of subdivision schemes. The blue $*$ denotes the original sampling of function (4.1), and the red solid line denotes the limit function

We consider the symmetric interpolatory scheme designed by Deslauriers and Dubuc in [28], when taking $m=2$ :

$$
\left\{\begin{array}{l}
f_{2 i}^{k+1}=f_{i}^{k}, \\
f_{2 i+1}^{k+1}=-\frac{1}{16} f_{i-1}^{k}+\frac{9}{16} f_{i}^{k}+\frac{9}{16} f_{i+1}^{k}-\frac{1}{16} f_{i+2}^{k} .
\end{array}\right.
$$

It produces the Gibbs phenomenon in the limit function close to discontinuity zones. In the numerical experiments we can clearly observe the Gibbs phenomenon, see Fig. 1(b).

Next, we will consider the ternary schemes.

We take the 3-point ternary subdivision scheme [29]:

$$
\left\{\begin{array}{l}
f_{3 i}^{k+1}=\frac{3}{8} f_{i-1}^{k}+\frac{1}{2} f_{i}^{k}+\frac{1}{8} f_{i+1}^{k} \\
f_{3 i+1}^{k+1}=\frac{3}{16} f_{i-1}^{k}+\frac{5}{8} f_{i}^{k}+\frac{3}{16} f_{i+1}^{k} \\
f_{3 i+2}^{k+1}=\frac{1}{8} f_{i-1}^{k}+\frac{1}{2} f_{i}^{k}+\frac{3}{8} f_{i+1}^{k}
\end{array}\right.
$$

Analogous to the binary schemes, according to Corollary 3.1, because of the positivity of the masks of the 3-point ternary scheme, it is easy to see that this scheme does not produce the Gibbs phenomenon; this result is shown in Fig. 1(c). 
We take the interpolating 5-point ternary subdivision scheme [30]:

$$
\left\{\begin{array}{l}
f_{3 i-1}^{k+1}=\left(\omega-\frac{4}{81}\right) f_{i-2}^{k}+\left(-4 \omega+\frac{10}{27}\right) f_{i-1}^{k}+\left(6 \omega+\frac{20}{27}\right) f_{i}^{k}-\left(4 \omega+\frac{5}{81}\right) f_{i+1}^{k}+\omega f_{i+2}^{k} \\
f_{3 i}^{k+1}=f_{i}^{k} \\
f_{3 i+1}^{k+1}=\omega f_{i-2}^{k}-\left(4 \omega+\frac{5}{81}\right) f_{i-1}^{k}+\left(6 \omega+\frac{20}{27}\right) f_{i}^{k}+\left(-4 \omega+\frac{10}{27}\right) f_{i+1}^{k}+\left(\omega-\frac{4}{81}\right) f_{i+2}^{k} .
\end{array}\right.
$$

For the interpolating 5-point ternary subdivision scheme, when $\omega=0$, it can produce Gibbs oscillations, see Fig. 1(d).

\section{Conclusions}

In this paper, we analyzed that a Gibbs phenomenon occurs for the $p$-ary subdivision schemes in the limit function close to discontinuity zones. A sufficient condition was presented to determine whether the $p$-ary subdivision scheme produces the Gibbs phenomenon close to discontinuities. This condition consists of the positivity of the partial sums of the values of the mask. By analyzing the properties of the masks of the subdivision schemes, we demonstrated how the Gibbs phenomenon can be removed from the $p$-ary subdivision schemes. Furthermore, in the numerical experiments we analyzed this phenomenon in classical subdivision schemes such as binary 5-point scheme, Deslauriers and Dubuc interpolation subdivision scheme, ternary 3-point, and ternary 5-point schemes. The numerical results validated that our results are efficient. In the future, we will focus on the Gibbs phenomenon and its resolution in other subdivision schemes, and we hope to give a common and uniform condition to remove this phenomenon.

Acknowledgements

We thank the editor and referees for their careful reading and valuable suggestions to make the article reader friendly

Funding

This work is supported by the Natural Science Basic Research Plan in Shaanxi Province of China (Program No. 2016JM6056); the National Natural Science Foundation of China (No. 71401134, 71571144). The authors thank all the reviewers for their careful reading and for their valuable comments.

\section{Competing interests}

The authors declare that they have no competing interests.

\section{Authors' contributions}

All authors contributed to each part of this work equally and read and approved the final manuscript.

\section{Publisher's Note}

Springer Nature remains neutral with regard to jurisdictional claims in published maps and institutional affiliations.

Received: 3 July 2018 Accepted: 11 February 2019 Published online: 26 February 2019

\section{References}

1. Amat, S., Ruiz, J., Trillo, J.C., Yáñez, D.F:: Analysis of the Gibbs phenomenon in stationary subdivision schemes. Appl. Math. Lett. 76, 157-163 (2018)

2. Gibbs, J.W.: Letter to the editor. Nature (London) 59, 606 (1899)

3. Michelson, A.A.: Letter to the editor. Nature 58, 544-545 (1898)

4. Carslaw, H.S.: A historical note on the Gibbs phenomenon in Fourier's series and integrals. Bull. Am. Math. Soc. 31, 420-424 (1925)

5. Mishra, L.N., Mishra, V.N., Khatri, K., Deepmala: On the trigonometric approximation of signals belonging to generalized weighted Lipschitz $W\left(L^{r}, \xi(t)\right)(r \geq 1)$-class by matrix $\left(C^{1} . N_{p}\right)$ operator of conjugate series of its Fourier series. Appl. Math. Comput. 237, 252-263 (2014)

6. Shim, H., Volkmer, H.: On the Gibbs phenomenon for wavelet expansions. J. Approx. Theory 84, 74-95 (1996)

7. Richards, F.B.: A Gibbs phenomenon for spline functions. J. Approx. Theory 66, 334-351 (1991)

8. Kelly, S.: Gibbs phenomenon for wavelets. Appl. Comput. Harmon. Anal. 3, 72-81 (1996)

9. Karanikas, C.: Gibbs phenomenon in wavelet analysis. Results Math. 34, 330-341 (1998) 
10. Ruch, D.K., Fleet, P.J.V.: Gibbs' phenomenon for nonnegative compactly supported scaling vectors. J. Math. Anal. Appl. 304(1), 370-382 (2005)

11. Shim, H.T., Kim, H.O.: On Gibbs' phenomenon for sampling series in wavelet subspaces. Appl. Anal. 61, 97-109 (1996)

12. Zhang, Z.H.: Convergence and Gibbs phenomenon of periodic wavelets frame series. Rocky Mt. J. Math. 39 , 1373-1396 (2009)

13. Ben, A., Anders, C.H.: Stable reconstructions in Hilbert spaces and the resolution of the Gibbs phenomenon. Appl. Comput. Harmon. Anal. 32, 357-388 (2012)

14. Amat, S., Dadourian, K., Liandrat, J.: On a nonlinear subdivision scheme avoiding Gibbs oscillations and converging towards $C^{s}$ function with s > 1. Math. Comput. 80(274), 959-971 (2011)

15. Jena, B.B., Mishra, L.N., Paikray, S.K., Misra, U.K.: Approximation of signals by general matrix summability with effects of Gibbs phenomenon. Bol. Soc. Parana. Mat. (2021). https://doi.org/10.5269/bspm.v38i6.39280

16. Mishra, V.N.: Some problems on approximations of functions in Banach spaces, Ph.D. Thesis, Indian Institute of Technology, Roorkee 247 667, Uttarakhand, India (2007)

17. Mishra, V.N., Mishra, L.N.: Trigonometric approximation of signals (functions) in $L_{p}(p \geq 1)$-norm. Int. J. Contemp. Math. Sci. 7(19), 909-918 (2012)

18. Mishra, V.N., Khatri, K., Mishra, L.N.: Deepmala: Trigonometric approximation of periodic signals belonging to generalized weighted Lipschitz $W^{\prime}\left(L_{r}, \xi(t)\right),(r \geq 1)$-class by Nörlund-Euler $\left(N, p_{n}\right)(E, q)$ operator of conjugate series of its Fourier series. J. Class. Anal. 5(2), 91-105 (2014)

19. Gottlieb, D., Shu, C.W.: On the Gibbs phenomenon and its resolution. SIAM Rev. 39(4), 644-668 (1997)

20. Jerri, A.J.: Lanczos-like $\sigma$-factors for reducing the Gibbs phenomenon in general orthogonal expansions and other representations. J. Comput. Anal. Appl. 2, 111-127 (2000)

21. Rim, K.S., Yun, B.I.: Gibbs phenomenon removal by adding Heaviside functions. Adv. Comput. Math. 38(4), 683-699 (2013)

22. Zhang, C.J., Wang, J.S.: An efficient de-noising algorithm for infrared image. In: Proceedings of the 2005 IEEE, pp. 513-517 (2005)

23. Zhou, X., Zhou, C.: Comparisons of discrete wavelet transform wavelet packet transform and stationary wavelet transform in denoising PD measurement date. In: Conference Record of the 2006 IEEE International Symposium on Electrical Insulation, pp. 237-240 (2006)

24. Dyn, N., Levin, D.: Subdivision schemes in geometric modelling. Acta Numer. 11 73-144 (2002)

25. Conti, C., Hormann, K.: Polynomial reproduction for univariate subdivision schemes of any arity. J. Approx. Theory 163, 413-437 (2011)

26. Dyn, N., Hormann, K., Sabin, M.A., Shen, Z.: Polynomial reproduction by symmetric subdivision schemes. J. Approx. Theory 155, 28-42 (2008)

27. Tan, J., Yao, Y., Cao, H., Zhang, L.: Convexity preservation of five-point binary subdivision scheme with a parameter Appl. Math. Comput. 245, 279-288 (2014)

28. Deslauriers, G., Dubuc, S.: Symmetric iterative interpolation processes. Constr. Approx. 5, 49-68 (1989)

29. Pitolli, F.: Ternary shape-preserving subdivision schemes. Math. Comput. Simul. 106, 185-194 (2014)

30. Novara, P., Romani, L:: On the interpolating 5-point ternary subdivision scheme: a revised proof of convexity-preservation and an application-oriented extension. Math. Comput. Simul. 147, 194-209 (2018)

\section{Submit your manuscript to a SpringerOpen ${ }^{\circ}$ journal and benefit from:}

- Convenient online submission

- Rigorous peer review

- Open access: articles freely available online

- High visibility within the field

- Retaining the copyright to your article

Submit your next manuscript at $\boldsymbol{~ s p r i n g e r o p e n . c o m ~}$ 